\title{
Population pharmacokinetic and pharmacodynamic properties of artesunate in patients with artemisinin sensitive and resistant infections in Southern Myanmar
}

Jesmin Permala Lohy Das ${ }^{1}$, Myat P. Kyaw ${ }^{2}$, Myat H. Nyunt ${ }^{2}$, Khin Chit ${ }^{2}$, Kyin H. Aye², Moe M. Aye ${ }^{2}$, Mats O. Karlsson ${ }^{1}$, Martin Bergstrand ${ }^{1}$ and Joel Tarning ${ }^{3,4^{*}}$

\begin{abstract}
Background: Artemisinins are the most effective anti-malarial drugs for uncomplicated and severe Plasmodium falciparum malaria. However, widespread artemisinin resistance in the Greater Mekong Region of Southeast Asia is threatening the possibility to control and eliminate malaria. This work aimed to evaluate the pharmacokinetic and pharmacodynamic properties of artesunate and its active metabolite, dihydroartemisinin, in patients with sensitive and resistant falciparum infections in Southern Myanmar. In addition, a simple nomogram previously developed to identify artemisinin resistant malaria infections was evaluated.

Methods: Fifty-three $(n=53)$ patients were recruited and received daily oral artesunate monotherapy $(4 \mathrm{mg} / \mathrm{kg})$ for 7 days. Frequent artesunate and dihydroartemisinin plasma concentration measurements and parasite microscopy counts were obtained and evaluated using nonlinear mixed-effects modelling.

Results: The absorption of artesunate was best characterized by a transit-compartment $(n=3)$ model, followed by one-compartment disposition models for artesunate and dihydroartemisinin. The drug-dependent parasite killing effect of dihydroartemisinin was described using an Emax function, with a mixture model discriminating between artemisinin sensitive and resistant parasites. Overall, $56 \%$ of the studied population was predicted to have resistant malaria infections. Application of the proposed nomogram to identify artemisinin-resistant malaria infections demonstrated an overall sensitivity of 90\% compared to $55 \%$ with the traditional day-3 positivity test.

Conclusion: The pharmacokinetic-pharmacodynamic properties of artesunate and dihydroartemisinin were wellcharacterized with a mixture model to differentiate between drug sensitive and resistant infections in these patients. More than half of all patients recruited in this study had artemisinin-resistant infections. The relatively high sensitivity of the proposed nomogram highlights its potential clinical usefulness.
\end{abstract}

Keywords: Malaria, Resistance, Parasite clearance, Artemisinin, Pharmacokinetics, Pharmacodynamics, Nonlinear mixed-effects modelling

\footnotetext{
*Correspondence: joel@tropmedres.ac

${ }^{3}$ Mahidol-Oxford Tropical Medicine Research Unit, Faculty of Tropical

Medicine, Mahidol University, Bangkok, Thailand

Full list of author information is available at the end of the article
} 


\section{Background}

Artemisinin-based combination therapy (ACT) is the recommended first-line therapy against uncomplicated Plasmodium falciparum malaria worldwide [1]. ACT is the most effective anti-malarial treatment today and consist of an artemisinin derivative and a partner drug [2]. The short-acting but potent artemisinin component eliminates the majority of parasites during the first 3 days of treatment and the slow acting and less potent partner drug removes residual parasites to prevent recrudescent infections. The introduction of ACT has contributed substantially to the reduction in malaria-related mortality and morbidity during the last decade [3].

However, the effectiveness of artemisinins is threatened in Southeast Asia due to widespread artemisinin resistance in the region [4]. Artemisinin resistant $P$. falciparum is defined clinically as delayed parasite clearance [5]. The clearance of microscopy detectable parasites by $24-48 \mathrm{~h}$ after the first dose of ACT is a typical indication of $P$. falciparum being fully susceptible to artemisinins [6], and parasite detection at $72 \mathrm{~h}$ after treatment initiation is interpreted as possible resistance [7]. Delayed parasite clearance results in higher parasite densities to be eliminated by the partner drug, causing an increased selection pressure for partner drug resistance [8]. High-grade resistance to both dihydroartemisinin and its partner drug, piperaquine, is now seen in Cambodia, Thailand, Laos and Vietnam resulting in unacceptably high clinical failure rates [9-11].

In early 2009, delayed parasite clearance was observed also in Myanmar [5, 12]. This was recently confirmed by the presence of slow parasite clearance as well as the in vivo molecular marker for artemisinin resistance (i.e. kelch 13 mutations) $[13,14]$. The fear is that this resistance genotype will spread through Bangladesh, India and find its way to the African continent similar to what was seen for chloroquine and sulfadoxine-pyrimethamine resistance [15].

Even though the discovery of kelch 13 marker for artemisinin resistance has changed the resistance monitoring paradigm, the day-3 positivity tests is still being used clinically to detect resistant malaria infections due to advantageous of cost, easy accessibility, and field applicability. However, the performance of this test is deemed rather ineffective as the absolute parasite clearance time, and therefore also day- 3 positivity, is very much dependent on the baseline parasite density [7]. The day-3 positivity test is particularly disadvantaged in high-endemic settings since acquired immunity often results in lower baseline parasitaemia and a faster parasite clearance rate. Thus, a new simple algorithm (baseline-adapted nomogram) which also takes into account the baseline density was proposed recently based on an analysis of data from a study in Thailand and Cambodia [4].
The primary aim of this study was to describe and evaluate the population pharmacokinetic and pharmacodynamic properties of artesunate (ARS) and its active metabolite, dihydroartemisinin (DHA), in the treatment of sensitive and resistant $P$. falciparum infections in Myanmar. A secondary aim was to perform an external validation of the recently proposed baseline-adapted nomogram to identify artemisinin resistant malaria infections.

\section{Methods \\ Study design}

This was a non-randomized, single arm, open-labelled clinical trial conducted in Palm Tree plantation site Hospital in Kawthaung in southern Myanmar. The trial was conducted to assess parasite clearance times in patients with uncomplicated $P$. falciparum malaria after ARS monotherapy. Clinical outcome and non-compartmental pharmacokinetic results have been published in full elsewhere (5). Ethical approval was obtained from the ethical review committees of the Department of Medical Research (Lower Myanmar), the Myanmar Ministry of Health, and the World Health Organization (WHO); Trial registration: Australian New Zealand Clinical Trials Registry ACTRN12610000896077.

Fifty-three $(n=53)$ patients who met all of the inclusion criteria and none of the exclusion criteria were recruited. Inclusion criteria; $18-55$ years old, monoinfection with $P$. falciparum, asexual parasite density of $10,000-100,000 / \mu \mathrm{l}$, fever in last $24 \mathrm{~h}$, ability to tolerate oral intake of ARS, agreement to comply with the study protocol, and provision of written informed consent. Exclusion criteria; severe malaria, severe malnutrition, pregnancy, lactation, mixed malaria infection, clinical evidence of infection other than malaria, history of chronic medical illness, splenectomy, hypersensitivity to ARS or related compounds, or reported use of drugs with anti-malarial activity within $48 \mathrm{~h}$ before enrollment.

Study drug was procured from Guilin Pharmaceutical Co. Ltd. (Shanghai, China); lot Number AS091001. All patients received directly observed oral ARS monotherapy $(4 \mathrm{mg} / \mathrm{kg} /$ day $)$ once daily for 7 days, administered with 8 oz. of milk.

Venous blood samples were taken immediately before and at $0.25,0.5,0.75,1.0,1.25,1.5,3,4,6$ and $8 \mathrm{~h}$ after the first dose. Within 15 min of collection, blood was centrifuged at $4{ }^{\circ} \mathrm{C}$ at $2000 \times g$ for $7 \mathrm{~min}$ and plasma was stored in liquid nitrogen until analysis. All samples were freighted on dry ice to the Department of Clinical Pharmacology at Mahidol-Oxford Tropical Medicine Research Unit (Bangkok, Thailand) where the plasma samples were analysed. The laboratory is a participant in the QA/QC proficiency testing programme supported by the Worldwide Antimalarial Resistance Network (WWARN) [16]. Plasma 
concentrations of ARS and DHA were measured by liquid chromatography-tandem mass spectroscopy using a published and validated method [17]. The observed total assay coefficients of variation was below $<8 \%$ in all quality control samples which were in concordance with FDA requirements, i.e. variations less than $15 \%$ at each low, medium and high concentration [18]. The lower limits of quantification (LLOQ) were 1.2 and $2.0 \mathrm{ng} / \mathrm{ml}$ for ARS and DHA, respectively. Microscopy parasite counts was performed every $12 \mathrm{~h}$ until two consecutive negative smears using Giemsa-stained thick and thin blood smears. Parasites were counted against 200 or 500 white blood cells and multiplied by an assumed white blood cell count of $6000 / \mu l$.

Population pharmacokinetic-pharmacodynamic modelling Data analysis was performed using nonlinear mixedeffects modelling implemented in the NONMEM software, v.7.3 (ICON Development Solutions, Ellicott City, MD) [19]. Xpose v.4.5.3 was used for graphical analysis and visual diagnostics of the model [20, 21]. PearlSpeaks-NONMEM (PsN), v. 4.5.5 [22], Xpose, Pirana, v. 2.9.2 (21), and R, v. 3.2.4 (The R Foundation for Statistical Computing) $[23,24]$ were used for other post-processing, model diagnostics, graphical analysis and automation.

Model selection was guided by plausible parameter estimates, precision of parameters, visual diagnostics and minimum objective function value (OFV) computed by NONMEM as proportional to minus twice the log likelihood of the data. A drop in OFV of 3.84 or more was considered a significant improvement $(\mathrm{p}<0.05)$ between two hierarchical models after inclusion of one additional parameter (one degree of freedom difference). Visual predictive checks (VPCs) were performed (2000 simulations) to evaluate the predictive performance of the pharmacokinetic and pharmacodynamic models. The reliability of individual parameter estimates and goodness-of-fit plots were assessed by evaluating eta and epsilon shrinkages. The $95 \%$ confidence intervals (CIs) of the estimated parameters and parameter uncertainties were calculated using the Sampling Importance Resampling (SIR) method [25, 26].

\section{Population pharmacokinetics}

Observed concentrations of ARS and DHA (molar units) were transformed into their natural logarithms and modelled simultaneously. Complete metabolic in vivo conversion of ARS into DHA was assumed throughout modelling [27]. Observations falling below the LLOQ was included and analysed using the Laplacian estimation method (i.e. previously established likelihood based M3-method) [29-30]. Models with one, two and three disposition compartments were explored and the best performing disposition model was carried forward to evaluate different absorption models, i.e. firstorder absorption with and without lag-time, zero-order absorption, sequential zero- and first-order absorption and transit-compartment absorption. The number of transit compartments was determined by stepwise addition of one to ten transit compartments to minimize the OFV. The drug transfer rate between transit compartments $\left(\mathrm{K}_{\mathrm{TR}}\right)$ was described by the equation below:

$$
K_{T R}=\frac{(n+1)}{M T T}
$$

where MTT is the mean transit time and $\mathrm{n}$ is the number of transit compartment.

Between subject variability (BSV) was modelled exponentially as described below:

$$
\boldsymbol{\theta}_{\boldsymbol{i}}=\boldsymbol{\theta}_{T V} \times \boldsymbol{e}^{\eta_{i}}
$$

where $\boldsymbol{\theta}_{\boldsymbol{i}}$ is the estimated individual parameter, $\boldsymbol{\theta}_{T V}$ is the estimated population parameter value, and $\boldsymbol{e}^{\eta_{i}}$ represents the BSV, assumed to be independent and normally distributed around zero with a variance $\omega^{2}$. The unexplained residual variability (RUV) was estimated by separate additive error models for log-transformed ARS and DHA concentrations (i.e. equal to exponential error models on an arithmetic scale).

Clearance and volume of distribution of both parent and metabolite were scaled allometrically using body weight. Scaled body weight was raised to the power of 0.75 and 1 for clearance and volume parameters, respectively, and centered on the median weight of the population.

Due to lack of subsequent concentration measurements after first dose, malaria disease effect as a covariate on the absorption rate (MTT) and relative bioavailability (F) was implemented a priori based on a previous analysis in a neighbouring region (Thailand-Cambodia) according to the equations below (i.e. Eq. 3 for MTT and Eq. 4 for F).

$$
\theta_{i}=\theta_{T V} \times\left(1+P A R A_{M T T} \times\left(\log \left(P A R A_{i}\right)-\log \left(P A R A_{\min }\right)\right)\right)
$$

$$
\theta_{i}=\theta_{T V} \times\left(1+\left[\frac{\left(\log \left(P A R A_{i}\right)-\log \left(P A R A_{\min }\right)\right) \times P A R A \max _{F}}{\left(\log \left(P A R A_{i}\right)-\log \left(P A R A_{\min }\right)\right)+\left(P A R A 50_{F}-\log \left(P A R A_{\min }\right)\right)}\right]\right)
$$


where PARA ${ }_{M T T}$ is the estimated linear effect of parasite density on MTT, PARAmax $x_{F}$ is the maximum effect of parasite density on F, PARA $50_{\mathrm{F}}$ is the parasite density which produces $50 \%$ of the maximum covariate response, and $\mathrm{PARA}_{\mathrm{i}}$ is the individual parasite density. All other potentially influential covariates (i.e. age, sex, baseline hemoglobin, and temperature) were evaluated on all parameters using a stepwise forward inclusion $(\mathrm{p}<0.05)$ and backward deletion $(\mathrm{p}<0.01)$ covariate modelling approach. Individual pharmacokinetic parameter estimates from the final pharmacokinetic model were imputed as posterior Bayes estimates into the pharmacokinetic-pharmacodynamic model.

\section{Population pharmacodynamics}

Total circulating parasite biomass was calculated by multiplying parasite counts by the individual patient's estimated blood volume, computed using Nadler's formula which takes into account gender, body weight and height [31]. Total parasite densities were transformed into their natural logarithms and modelled initially using a simplified one-compartment parasite model [32]. Data below the LLOQ (less than 12 parasites per $\mu \mathrm{l}$ ) were modelled with the M3-method [28, 30]. Parasites were assumed to have a tenfold multiplication per asexual cycle of $48 \mathrm{~h}$ [33]. Drug effects were evaluated using a direct response model (i.e. basic Emax model and sigmoidal Emax model) and an indirect response model (i.e. delayed effect model/ hysteresis). Individually predicted plasma concentrations of DHA were used to evaluate the drug-dependent killing of parasites (KILL), leading to an approximate log-linear reduction in parasite numbers with time (Eq. 5).

$$
K I L L=\frac{E_{\max } \times C e}{E C_{50}+C e}
$$

where Emax is the maximum parasite killing effect, $\mathrm{Ce}$ is the DHA drug concentration in the effect compartment and $\mathrm{EC}_{50}$ is the concentration which produces $50 \%$ of maximum killing effect. A mixture model to identify artemisinin resistant and artemisinin sensitive parasite infections was implemented on Emax, and the probability of having an artemisinin resistant infection $(\mathbf{M I X i}=2)$ was estimated on a logit-transformed domain (Eq. 6).

$$
\log \text { it }[\mathrm{P}(\mathrm{MIXi}=2)]=\ln \left(\frac{\theta_{\mathrm{PMIX} 2_{\text {resistant }}}}{1-\theta_{\mathrm{PMIX}} 2_{\text {resistant }}}\right)
$$

where $\boldsymbol{\theta}_{\mathbf{P M I X}} \mathbf{2}_{\text {resistant }}$ is the population probability of belonging to mixture 2 (artemisinin resistant infection). Individual Emax-values associated with an artemisinin sensitive infection (higher parasite killing effect) and artemisinin resistant infection were parameterized as below to improve stability, and to constrain all individual $\operatorname{Emax}_{\mathrm{Si}}$ values to be greater than the typical population Emax value associated with a resistant infection [34].

$$
\begin{aligned}
& \boldsymbol{E m a x}_{S i}=\boldsymbol{\theta}_{T V R}+\left(\boldsymbol{\theta}_{T V S}-\boldsymbol{\theta}_{T V R}\right) \times \boldsymbol{e}^{\eta_{S}} \\
& \boldsymbol{E}_{\max _{\boldsymbol{R} i}}=\boldsymbol{\theta}_{T V R} \times \boldsymbol{e}^{\eta_{R}}
\end{aligned}
$$

where $\boldsymbol{\theta}_{\mathrm{TVR}}$, and $\boldsymbol{\theta}_{\mathrm{TVS}}$ are the estimated typical parameter values for resistant infections and sensitive infections, respectively, with their variance $(\eta)$. A frequentist prior functionality [35] was implemented a priori to support the estimation of $\mathrm{EC}_{50}$ and $\mathrm{Emax}$ in patients with artemisinin sensitive infections. The $\mathrm{EC}_{50}$ and Emax estimates from the artemisinin sensitive subgroup in a previous analysis of patients from Thailand and Cambodia $\left(\mathrm{EC}_{50}: 34.9 \mathrm{nM}\right.$ [31.7\% RSE] and Emax: $0.273 \mathrm{~h}^{-1}$ [6.25\% RSE]) were used for this purpose. This was based on the assumption that the drug sensitivity in the nonresistant population is likely to be similar in the greater Mekong sub region.

\section{Evaluation of the resistance nomogram}

A simple diagnostic method to detect resistant malaria infections was recently developed, based on data from Thailand and Cambodia [36]. This new diagnostic method demonstrated to be a promising alternative to the current practice of day- 3 positivity test as a proxy of having a drug resistant malaria infection [36]. The developed nomogram used baseline parasite counts to select the time point post-treatment to assess the parasite count. These post-treatment parasite counts in relation to baseline parasite density were used to identify patients with resistant infections.

The nomogram was applied in this current dataset to act as external validation of the proposed method. The individual parasite ratio $\left(\right.$ Ratio $\left._{i}\right)$ of baseline parasite count and that at the chosen assessment time point $j$ (i.e. 24,48 or $72 \mathrm{~h}$ post-treatment) was calculated as below (Eq. 9)

$$
\text { Ratio }_{i, j(24,48,72)}=\log (P A R A)_{i, 0}-\log (P A R A)_{i, j(24,48,72)}
$$


The nomogram-suggested "cut-off" value for chosen time points (i.e. 1.46 for day 1 [baseline parasite density $10^{9}-10^{10.5}$ ], 2.93 for day 2 [baseline parasite density $\left.10^{10.5}-10^{12}\right]$ and 4.34 for day- 3 assessment $\left[>10^{12}\right]$ ) was applied on the Ratio ${ }_{i}$. If the Ratio $_{i}$ value was below the "cut-off" value, the patient was classified to have an artemisinin resistant infection as below.

$$
\text { Classification }=\left\{\begin{array}{l}
\text { Resistant }, \text { (if Ratio } \text { R }_{i}<\text { "cut-off") } \\
\text { Sensitive },(\text { if Ratio } i>\text { "cut-off") }
\end{array}\right.
$$

Whenever the parasite density value at the proposed assessment day was below LLOQ, the patient was directly classified as having an artemisinin sensitive infection and the ratio calculation was not performed. This classification was compared to patient's mixture assignment from the model probability estimates, which was regarded as the "truth". A sensitivity analysis was performed on this comparison and the performance of the nomogram was evaluated.

Table 1 Baseline study demographics

\begin{tabular}{lc}
\hline Characteristics & Median (interquartile range) \\
\hline Weight $(\mathrm{kg})$ & $50.0(46.0-53.5)$ \\
Age (years) & $25.5(21.5-39.5)$ \\
Oral temperature at enrollment $\left({ }^{\circ} \mathrm{C}\right)$ & $38.4(37.6-39.1)$ \\
Haemoglobin $(\mathrm{g} / \mathrm{dl})$ & $12.4(10.6-13.7)$ \\
Baseline parasite density $($ parasite/ $\mu \mathrm{l})$ & $29,900(15,200-129,000)$ \\
Fever clearance time $($ day) & $3(2-4)$ \\
\hline
\end{tabular}

\section{Results}

A total of 53 patients were recruited to the study and 1 patient was excluded from the study for not meeting the inclusion/exclusion criteria. Two patients were excluded from the pharmacokinetic analysis since they were missing important covariates as well as pharmacokinetic and pharmacodynamic data. Baseline characteristics for the studied population are presented in the Table 1.

\section{Population pharmacokinetics}

The population pharmacokinetic properties of ARS and DHA were best described using a single disposition compartment model for each of the drug molecules (Fig. 1). The absorption was described by a transit compartment $(\mathrm{n}=3)$ model, which was superior to other absorption models. Allometric scaling of all disposition parameters, centered by the median weight of $50 \mathrm{~kg}$ improved the model fit. Malaria disease was implemented a priori as a time-varying covariate on MTT and F, generating a decreased MTT and increased F with increasing parasite counts. No other covariates had a significant impact on the pharmacokinetic parameters in the final model. BSV was maintained in all parameters and the eta shrinkages computed in the final pharmacokinetic model were moderate to low $\left(\mathrm{CL}_{\mathrm{ARS}}=36.6 \%, \mathrm{~V}_{\mathrm{ARS}}=14.8 \%\right.$, $\mathrm{CL}_{\mathrm{DHA}}=38.6 \%, \quad \mathrm{~V}_{\mathrm{DHA}}=32.4 \%, \quad \mathrm{MTT}=7.01 \% \quad$ and $\mathrm{F}=20.2 \%)$ while epsilon shrinkages were low $(14.1 \%$ for ARS and $10.0 \%$ for DHA). Simulation-based diagnostics (i.e. VPC) showed satisfactory predictive performance of the final pharmacokinetic model describing ARS and DHA (Fig. 2). Final population pharmacokinetic

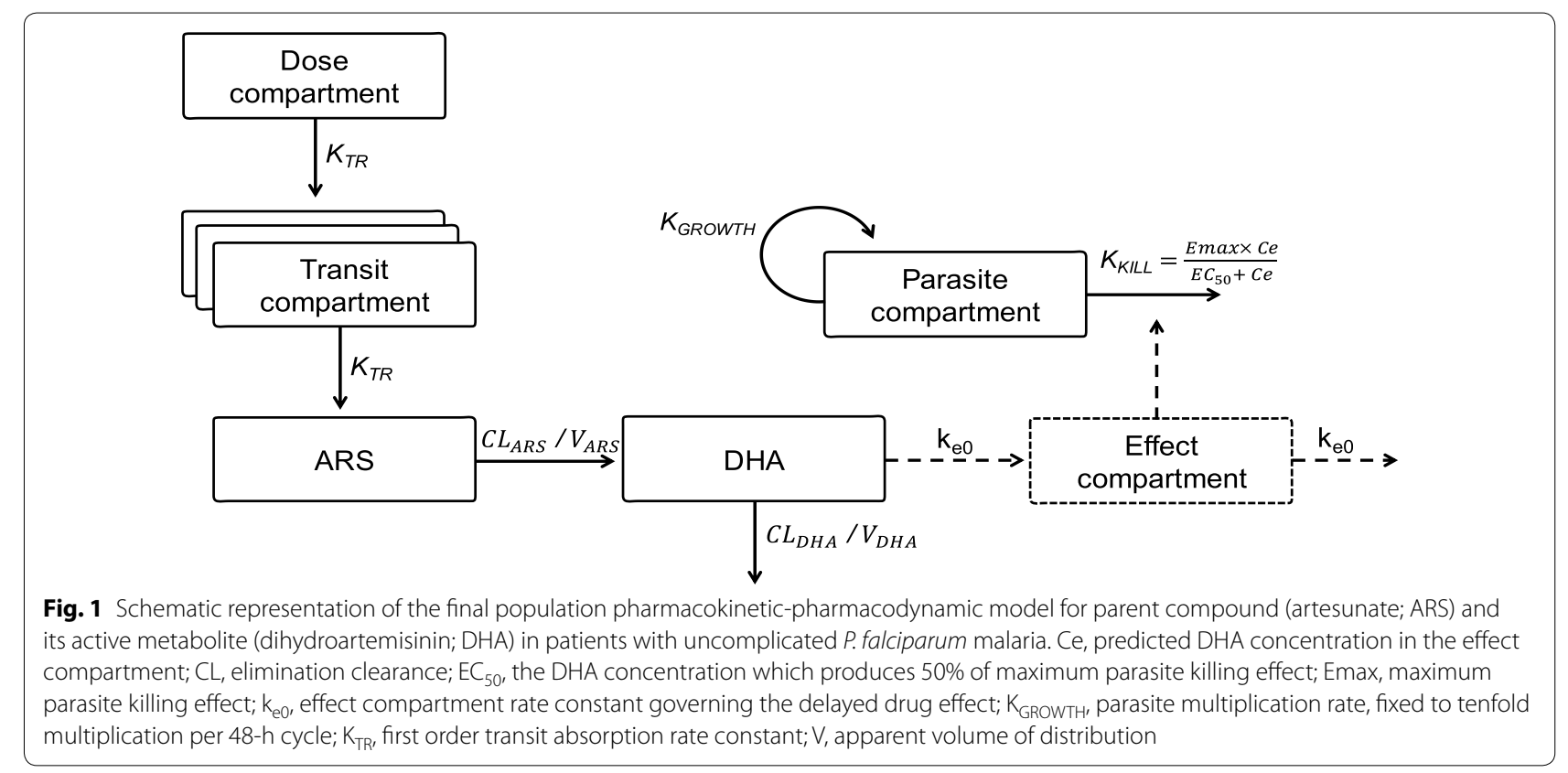




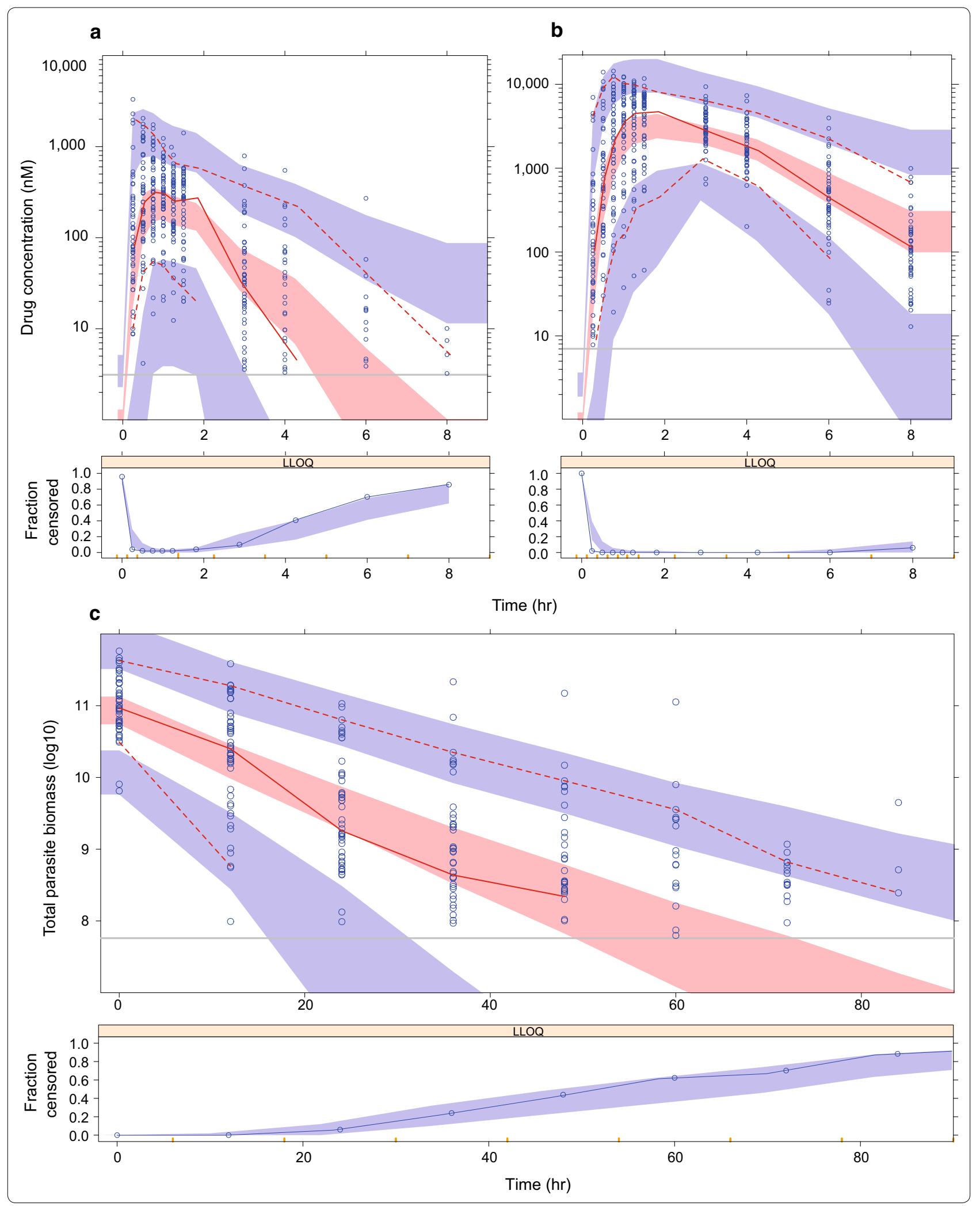


(See figure on previous page.)

Fig. 2 Visual predictive check of final population pharmacokinetic model of artesunate (a) and dihydroartemisinin (b), and population pharmacodynamic model (c) in patients with uncomplicated P. falciparum malaria. The open circles are observed data points, the solid red line represents the 50th percentile of observed data; dashed red lines represent the 5th and 95th percentiles (pharmacokinetic model) and the 10th and 90th percentiles (pharmacodynamic model) of observed data; shaded areas are the model predicted $95 \%$ confidence intervals of the simulated percentiles; vertical grey lines represent the lower limit of quantification (LLOQ) for artesunate (3.12 nM), dihydroartemisinin (7.02 nM) and parasite density $\left(10^{7.73}\right.$ ). The lower panels show the fraction of observed data below the LLOQ (open circles) overlaid with the $95 \%$ prediction interval of the fraction of simulated data below the LLOQ (shaded area)

parameter estimates for ARS and DHA are presented in Table 2.

\section{Population pharmacodynamics}

The final pharmacokinetic-pharmacodynamic model is illustrated in Fig. 1. A delayed effect model showed a significantly better model fit compared to a direct effect model, and resulted in a half-life of the effect delay of $5.64 \mathrm{~h}$. A mixture model implementation on Emax (to distinguish the parasite clearance between resistant and sensitive parasites), resulted in a better model fit compared to when implementing the mixture on $\mathrm{EC}_{50}$, with no additional benefit on having a mixture on both parameters. Sensitive infections were characterized by an Emax

Table 2 Parameter estimates of the final pharmacokinetic-pharmacodynamic model

\begin{tabular}{|c|c|c|c|c|}
\hline Parameter & Estimates (\% RSE) & $95 \% \mathrm{Cl}$ & \%CV BSV (\% RSE) & $95 \% \mathrm{Cl}$ \\
\hline \multicolumn{5}{|l|}{ Pharmacokinetics } \\
\hline \multicolumn{5}{|l|}{ Artesunate } \\
\hline$F(\%)$ & $100 f i x$ & - & $31.2(29.4)$ & $19.3-50.8$ \\
\hline MTT (h) & $1.34(18.8)$ & $1.04-1.96$ & $85.3(24.9)$ & $65.7-133.0$ \\
\hline $\mathrm{CL}_{\mathrm{ARS}} / \mathrm{F}(\mathrm{I} / \mathrm{h})$ & $1750(8.55)$ & $1570-2090$ & $26.8(44.3)$ & $11.9-39.1$ \\
\hline $\mathrm{V}_{\mathrm{ARS}} / \mathrm{F}(\mathrm{I})$ & $1300(12.6)$ & $1110-1660$ & $74.7(27.3)$ & $57.8-129$ \\
\hline RUV (\%) & $73.2(3.95)$ & $69.3-78.7$ & - & - \\
\hline \multicolumn{5}{|l|}{ Dihydroartemisinin } \\
\hline $\mathrm{CL}_{\mathrm{DHA}} / \mathrm{F}(\mathrm{I} / \mathrm{h})$ & $76.7(6.99)$ & $69.9-87.8$ & $21.3(30.3)$ & $13.3-88.1$ \\
\hline $\mathrm{V}_{\mathrm{DHA}} / \mathrm{F}(\mathrm{I})$ & $102.0(8.95)$ & $89.5-119.0$ & $31.6(40.5)$ & $21.3-131.0$ \\
\hline RUV (\%) & $58.5(3.34)$ & $56.6-63.4$ & - & - \\
\hline \multicolumn{5}{|l|}{ Covariate effects } \\
\hline${ }^{a^{P} A R A}{ }_{M T T}(\log 10$ parasitaemia) & $0.115(8.88)$ & $0.121-0.156$ & - & - \\
\hline${ }^{a P A R A m a x} x_{F}$ & $1.51(11.9)$ & $1.35-2.02$ & - & - \\
\hline${ }^{\text {apARA50 }}$ (Log10 parasitaemia) & $8.32(3.58)$ & $8.19-9.21$ & - & - \\
\hline \multicolumn{5}{|l|}{ Pharmacodynamics } \\
\hline $\mathrm{K}_{\mathrm{GROWTH}}\left(48 \mathrm{~h}^{-1}\right)$ & 10 fix & & - & \\
\hline BASE $_{\text {PARA }}\left(\log _{10}\right)$ & $11.0(0.704)$ & $10.8-11.1$ & $4.4(19.6)$ & $3.13-5.78$ \\
\hline $\mathrm{k}_{\mathrm{e} 0}\left(\mathrm{~h}^{-1}\right)$ & $0.123(33.1)$ & $0.0584-0.188$ & - & \\
\hline${ }^{a} \mathrm{EC}_{50}(\mathrm{nM})$ & $30.4(34.2)$ & $13.5-46.1$ & - & \\
\hline${ }^{a} \operatorname{Emax}_{S}\left(h^{-1}\right)$ & $0.268(5.89)$ & $0.242-0.295$ & $b_{49.0}(22.4)$ & $34.3-70.1$ \\
\hline $\operatorname{Emax}_{R}\left(h^{-1}\right)$ & $0.155(6.08)$ & $0.142-0.172$ & $12.2(45.5)$ & $6.54-35.8$ \\
\hline$P_{M I X, \text { resistant }}(\%)$ & $56.1(20.9)$ & $39.1-73.8$ & - & \\
\hline RUV (\%) & $33.3(5.91)$ & $30.5-37.1$ & - & \\
\hline
\end{tabular}

Coefficient of variation (\%CV) of between subject variability (BSV) was calculated as $100 \times\left(\right.$ variance-1) ${ }^{1 / 2}$. Relative standard errors (\% RSE) were calculated as $100 \times$ (standard deviation/mean). The $95 \%$ confidence intervals $(95 \% \mathrm{CI}$ ) of parameter estimates were obtained with the Sampling Importance Resampling (SIR) approach

$A R S$ artesunate, $B A S E_{P A R A}$ baseline parasitaemia, $C L$ clearance, $D H A$ dihydroartemisinin, $F$ bioavailability, $K_{G R O W T H}$ parasite multiplication per $48 \mathrm{~h}$ parasite cycle, $M T T$

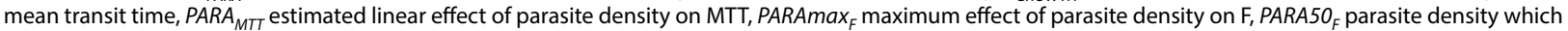
produces $50 \%$ of the maximum covariate response, $P_{M I X}$, resistant probability of having an artemisinin-resistant infection, $V$ volume of distribution, $E C_{50}$ the $\mathrm{DHA}$ concentration which produces $50 \%$ of maximum parasite killing effect, Emax ${ }_{R}$ maximum parasite killing effect of a resistant parasite population, Emax maximum parasite killing effect of a sensitive parasite population, $k_{e 0}$ effect compartment rate constant governing the delayed drug effect, $R U V$ unexplained residual variability

a Estimation of these parameters were obtained by applying a frequentist prior approach using a previously published PK/PD model developed on data from Thailand and Cambodia (37)

b BSV (\%CV) of Emax sas calculated based on simulations (10,000 patients) with an estimated variance of 0.430 and the applied transformation presented in Eq. 7 
estimate of $0.268 \mathrm{~h}^{-1}$ (5.89\% RSE) compared to $0.155 \mathrm{~h}^{-1}$ (6.08\% RSE) for the resistant infections, with an estimated $56.1 \%$ of patients having a resistant infection. $\mathrm{EC}_{50}$ was estimated to $8.64 \mathrm{ng} / \mathrm{ml}(30.4 \mathrm{nM})$ but with a relatively high uncertainty (34.2\% RSE). There were no significant covariates in the final pharmacodynamic model. The eta shrinkage was 35.0, 18.0 and $27.0 \%$ for Emax (sensitive), baseline and Emax (resistant) parameters, respectively, while epsilon shrinkage was $6 \%$. The final pharmacokinetic-pharmacodynamic model parameters and VPCs are presented in Table 2 and Fig. 2.

\section{Resistance nomogram}

Data from the current analysis were used as external validation for a recently developed nomogram from a study in Thailand and Cambodia $[4,36]$. Applying the baselineadapted nomogram to identify patients with resistant parasite infections, resulted in $90.1 \%$ overall sensitivity and $92.1 \%$ overall accuracy compared to 55.1 and $75.2 \%$, respectively, using the traditional day-3 positivity test. Complete performance of the baseline-adapted nomogram in comparison to the currently used day-3 positivity test is presented in Table 3.

\section{Discussion}

The spread of artemisinin resistance is threatening the effectiveness of artemisinin-based combination therapies, and it is crucial to monitor the spread and

Table 3 Predictive performance of the baseline-adapted nomogram and the day- 3 positivity test

\begin{tabular}{|c|c|c|c|}
\hline Statistics metric & $\begin{array}{l}\text { Baseline-adapted } \\
\text { nomogram }\end{array}$ & & Day-3 positivity test \\
\hline Negative results (N) & $\begin{array}{l}\text { The nomogram predi } \\
\text { the individual paras } \\
\text { density ratio (Ratio ) } \\
\text { above the "Cut-off" }\end{array}$ & $\begin{array}{l}\text { cts } \\
\text { ite } \\
\text { to be }\end{array}$ & $\begin{array}{l}\text { Non-resistant if } \\
\text { observed parasitae- } \\
\text { mia is below LLOQ } \\
\text { at day } 3\end{array}$ \\
\hline Positive results $(P)$ & $\begin{array}{l}\text { The nomogram predi } \\
\text { the individual paras } \\
\text { density ratio (Ratioj) } \\
\text { below the "Cut-off" }\end{array}$ & $\begin{array}{l}\text { cts } \\
\text { ite } \\
\text { to be }\end{array}$ & $\begin{array}{l}\text { Resistant if observed } \\
\text { parasitaemia is } \\
\text { above LLOQ at } \\
\text { day } 3\end{array}$ \\
\hline True positive (TP) & \multicolumn{3}{|c|}{$\begin{array}{l}\text { The approach predicts correctly the patient to } \\
\text { have a resistant infection }\end{array}$} \\
\hline True negative (TN) & \multicolumn{3}{|c|}{$\begin{array}{l}\text { The approach predicts correctly the patient to } \\
\text { have a sensitive infection }\end{array}$} \\
\hline Sensitivity $\frac{T P}{T P+F N}$ & $\begin{array}{l}\text { Probability of pre- } \\
\text { dicting correctly } \\
\text { patients with } \\
\text { resistant infections }\end{array}$ & $90 \%$ & $55 \%$ \\
\hline Specificity $\frac{T N}{T P+F P}$ & $\begin{array}{l}\text { Probability of pre- } \\
\text { dicting correctly } \\
\text { patients with } \\
\text { sensitive infections }\end{array}$ & $95 \%$ & $95 \%$ \\
\hline $\begin{array}{l}\text { Accuracy } \\
\frac{T P+T N}{T P+T N+F P+F N}\end{array}$ & $\begin{array}{l}\text { Proportion of all cor- } \\
\text { rect predictions }\end{array}$ & $93 \%$ & $75 \%$ \\
\hline
\end{tabular}

development of artemisinin resistance in Southeast Asia and elsewhere.

The final population pharmacokinetic model of ARSDHA was similar to that recently developed on data from a study conducted in Thailand and Cambodia [36]. The absorption of ARS was described using a transit compartment absorption model followed by a single distribution compartment for both ARS and DHA. The estimated population pharmacokinetic parameters were in good agreement with that estimated in previous studies, except for $V_{A R S}$, which was somewhat larger in this study [27, 37, 38]. Data presented here cannot elucidate the reason for potential systematic differences but it is well known that ARS is unstable and can rapidly undergo ex vivo conversion to DHA if not collected optimally [16, 39]. Thus, potential differences might be due to slightly different collection procedures in different studies. It was not surprising that body weight had a significant impact on pharmacokinetic parameters, considering that most physiological parameters scale by body weight. In addition, the malaria disease effect on absorption parameters (MTT and F) described in the previous study from Thailand and Cambodia [36] was implemented a priori. The current study featured plasma concentration data from the first administered dose only and the effect of declining parasite densities (i.e. malaria disease effect) on pharmacokinetic parameters could not have been modeled beyond the first dose. Inclusion of this effect based on prior information was incorporated to prevent bias in the drug potency estimate $\left(\mathrm{EC}_{50}\right)$ since the pharmacokinetic model would have otherwise predicted higher plasma concentrations beyond the first dose.

The pharmacokinetic-pharmacodynamic model developed here described the observed parasite data well. A delayed effect model was superior to a direct effect model, and resulted in a half-life of the effect delay of almost $6 \mathrm{~h}$, which is somewhat shorter to the $10 \mathrm{~h}$ described previously in Cambodia and Thailand [36]. The estimated delayed effect might reflect a delayed removal of injured and/or dead parasites, which contributes to an apparent sustained killing of parasites beyond the dosing interval. A frequentist prior based on the Thailand-Cambodia study was included to support the estimation of $\mathrm{EC}_{50}$ and the artemisinin sensitive Emax value. Prior information was deliberately not applied for other model parameters such as the artemisinin resistant Emax value since resistance could differ both between regions and over time [40]. However, the results indicated rather similar estimates of resistant infections in Myanmar (performed in year 2011; $\operatorname{Emax}=0.155$, RSE $6.08 \%$ ) and Thailand-Cambodia (performed in year 2007; Emax $=0.187$, RSE $4.88 \%$ ). This is in line with a recent molecular genotyping study that demonstrated that the 
artemisinin resistant genotype appears to have spread mostly throughout the region from 2008 to recent years (2014-2015), rather than developed regionally [11].

A total of $56 \%$ of patients recruited in this study was estimated to have artemisinin resistant infections, demonstrating a high prevalence of artemisinin resistance in the region. However, the original parasite clearance analysis of these data reported that 19 out of 52 (36.5\%) patients in this study had microscopy detectable parasite densities on day 3 (i.e. day-3 positivity test), which is substantially lower than the model-based analyses (5). This is in line with findings from a previous study in Thailand-Cambodia that also suggested that the day- 3 positivity test underestimates the number of artemisinin resistant infections compared to a model-based analysis. A likely explanation could be the inability of the day- 3 positivity test to take into account the impact of baseline parasite biomass.

The baseline-adapted nomogram was developed based on the relationship that the baseline parasite density is directly proportional to the parasite clearance time, assuming similar drug dependent elimination of parasites [36]. The presented nomogram was suggested to perform better than the commonly used day-3 positivity test, and to have high sensitivity in identifying patients with artemisinin resistant infections [36]. External validation of the nomogram using the data collected in this study in Myanmar showed an overall performance of $90 \%$ sensitivity and accuracy, exceeding the expectations of $80 \%$ sensitivity and accuracy presented previously [36]. This can be attributed primarily to few patients with low baseline parasite densities (i.e. below $10^{10.5}$ parasites were the nomogram is known to be less accurate). The reference test of day-3 positivity demonstrated similar inadequate sensitivity (55\%) and overall accuracy (75\%) as previously concluded. The developed baseline-adapted nomogram offered a high overall accuracy, but primarily a better sensitivity to identify artemisinin resistant malaria infections compared to the traditional day- 3 analysis. It also provides a simplified and field-adapted identification of resistant infections by using only one parasite measurement post-dosing. However, the use of the nomogram requires further validation in different epidemiological settings, in adults and children with different levels of immunity as well as different kelch 13 mutations.

The high prevalence of artemisinin resistance found in this study supports the concern that artemisinin resistance is spreading in the Greater Mekong sub-region. A widespread resistance to artemisinins could potentially reverse the positive trend of recent years of declining morbidity and mortality from malaria [1]. Therefore, it is imperative that containment efforts are scaled-up throughout Myanmar to stop the artemisinin resistance from spreading. In areas where artemisinin resistance is already prevalent, appropriate actions needs to be taken to mitigate the effects, especially the impact on selection for partner drug resistance. Prolonged artemisinin treatment from 3 to 5 days or administration of triple artemisininbased combinations has been proposed as potential interventions to reduce the pressure on the partner drug and thus combat artemisinin resistance [41, 42].

\section{Conclusion}

The pharmacokinetic-pharmacodynamic model developed here was able to describe the concentration-effect relationship of ARS and DHA in southern Myanmar. The model was able to confirm a high level of artemisinin resistance in this region. Urgent containment efforts and clinical and parasitological monitoring should remain a high priority [43]. In addition, the predictive value of a simple baseline-adapted nomogram for identification of artemisinin-resistant infections was evaluated and outperformed the traditionally used day-3 positivity test and supports its implementation in clinical use.

\section{Authors' contributions}

JPLD, MB and JT performed the pharmacometric analysis. MPK, MHN, KC, KHA and MMA collected the clinical data. JT was responsible for the drug measurements. JPLD, MB and JT wrote the first draft of the manuscript. All authors read and approved the final manuscript.

\section{Author details \\ ${ }^{1}$ Department of Pharmaceutical Biosciences, Uppsala University, Uppsala, Sweden. ${ }^{2}$ Department of Medical Research, Yangon, Republic of the Union of Myanmar. ${ }^{3}$ Mahidol-Oxford Tropical Medicine Research Unit, Faculty of Tropical Medicine, Mahidol University, Bangkok, Thailand. ${ }^{4}$ Centre for Tropi- cal Medicine, Nuffield Department of Clinical Medicine, University of Oxford, Oxford, UK.}

\section{Acknowledgements}

We sincerely thank all the patients for their cooperation in completing this study. We also thank the medical, nursing, and administrative staff at Kawthaung in southern Myanmar, and laboratory staff at Mahidol-Oxford Tropical Medicine Research Unit in Bangkok. The Mahidol-Oxford Tropical Medicine Research Unit is supported by the Wellcome Trust of Great Britain. The trial was also supported by grants from the Bill and Melinda Gates Foundation.

\section{Competing interests}

The authors declare that they have no competing interests.

\section{Availability of data and materials}

The datasets used and/or analysed during the current study are available from the corresponding author on reasonable request.

\section{Ethics approval and consent to participate}

Ethical approval for this study was granted from the Department of Medical Research (Lower Myanmar), the Myanmar Ministry of Health, and the WHO. Written informed consent was provided by all study participants. The trial was registered under Australian New Zealand Clinical Trials Registry (ACTRN12610000896077).

\section{Publisher's Note}

Springer Nature remains neutral with regard to jurisdictional claims in published maps and institutional affiliations.

Received: 13 November 2017 Accepted: 15 March 2018

Published online: 23 March 2018 


\section{References}

1. WHO. World malaria report 2016. Geneva: World Health Organization; 2016.

2. WHO. Guidelines for the treatment of malaria. Geneva: World Health Organization; 2015

3. WHO. World malaria report 2015. Geneva: World Health Organization; 2015

4. Dondorp AM, Nosten F, Yi P, Das D, Phyo AP, Tarning J, et al. Artemisinin resistance in Plasmodium falciparum malaria. $N$ Engl J Med. 2009;361:455-67.

5. Kyaw MP, Nyunt MH, Chit K, Aye MM, Aye KH, Aye MM, et al. Reduced susceptibility of Plasmodium falciparum to artesunate in southern Myanmar. PLOS ONE. 2013:8:e57689.

6. White NJ. The parasite clearance curve. Malar J. 2011;10:278.

7. Stepniewska K, Ashley E, Lee SJ, Anstey N, Barnes KI, Binh TQ, et al. In vivo parasitological measures of artemisinin susceptibility. J Infect Dis. 2010;201:570-9.

8. Noedl H, Se Y, Schaecher K, Smith BL, Socheat D, Fukuda MM. Evidence of artemisinin-resistant malaria in western Cambodia. N Engl J Med. 2008:359:2619-20.

9. Thanh NV, Thuy-Nhien N, Tuyen NT, Tong NT, Nha-Ca NT, Dong LT, et al. Rapid decline in the susceptibility of Plasmodium falciparum to dihydroartemisinin-piperaquine in the south of Vietnam. Malar J. 2017;16:27.

10. Phuc BQ, Rasmussen C, Duong TT, Dong LT, Loi MA, Menard D, et al. Treatment failure of dihydroartemisinin/piperaquine for Plasmodium falciparum malaria, Vietnam. Emerg Infect Dis. 2017;23:715-7.

11. Imwong M, Suwannasin K, Kunasol C, Sutawong K, Mayxay M, Rekol H, et al. The spread of artemisinin-resistant Plasmodium falciparum in the Greater Mekong subregion: a molecular epidemiology observational study. Lancet Infect Dis. 2017;17:491-7.

12. WHO. Workshop to review and plan therapeutic efficacy studies to monitor $P$. falciparum and $P$. vivax resistance to anti-malarial drugs in the Greater Mekong Sub-region; May 3, 2012.

13. Ashley EA, Dhorda M, Fairhurst RM, Amaratunga C, Lim P, Suon S, et al. Spread of artemisinin resistance in Plasmodium falciparum malaria. N Engl J Med. 2014;371:411-23.

14. Phyo AP, Ashley EA, Anderson TJC, Bozdech Z, Carrara VI, Sriprawat K, et al. Declining efficacy of artemisinin combination therapy against $P$. falciparum malaria on the Thai-Myanmar border (2003-2013): the role of parasite genetic factors. Clin Infect Dis. 2016;63:784-91

15. Dondorp AM, Yeung S, White L, Nguon C, Day NPJ, Socheat D, et al. Artemisinin resistance: current status and scenarios for containment. Nat Rev Microbiol. 2010;8:272-80.

16. Lourens C, Lindegardh N, Barnes Kl, Guerin PJ, Sibley CH, White NJ, et al. Benefits of a pharmacology antimalarial reference standard and proficiency testing program provided by the Worldwide Antimalarial Resistance Network (WWARN). Antimicrob Agents Chemother 2014;58:3889-94.

17. Lindegårdh N, Dondorp AM, Singhasivanon P, White NJ, Day NPJ. Validation and application of a liquid chromatographic-mass spectrometric method for determination of artesunate in pharmaceutical samples. Pharm Biomed Anal. 2007;45:149-53.

18. U.S. Department of Health and Human Services Food and Drug Administration, Center for Drug Evaluation and Research (CDER), Center for Veterinary Medicine (CVM). Guidance for Industry: Bioanalytical Method Validation. FDA, Maryland; 2001.

19. NONMEM 7: Beal S, Sheiner LB, Boeckmann A, Bauer RJ. NONMEM User's Guides. (1989-2009). Ellicott City: Icon Development Solutions; 2009.

20. Hooker AC, Karlsson MO, Wilkins JJ, Jonsson EN. Xpose4: tools for nonlinear mixed-effect model building and diagnostics. R package version 4.5.3. 2014

21. Keizer RJKM, Hooker AC. Modeling and simulation workbench for NONMEM: tutorial on Pirana, PSN, and Xpose. CPT Pharmacometrics Syst Pharmacol. 2013:2:e50.
22. Lindbom L, Pihlgren P, Jonsson EN. PsN-Toolkit-a collection of computer intensive statistical methods for non-linear mixed effect modeling using NONMEM. Comput Methods Programs Biomed. 2005;79:241-57.

23. R Core Team. R. A language and enviroment for statistical computing. Vienna: R Foundation for Statistical Computing; 2013.

24. Wickham WCH. ggplot2: elegant graphics for data analysis. New York: Springer; 2009

25. Dosne A-G, Bergstrand M, Harling K, Karlsson MO. Improving the estimation of parameter uncertainty distributions in nonlinear mixed effects models using sampling importance resampling. J Pharmacokinet Pharmacodyn. 2016;43(6):583-96. https://www.ncbi.nlm.nih.gov/pmc/ articles/PMC5110709/

26. Dosne A-G, Bergstrand M, Karlsson MO. Application of sampling importance resampling to estimate parameter uncertainty distributions; 2013. p. 22. https://www.page-meeting.org/?abstract $=2907$.

27. McGready R, Phyo AP, Rijken MJ, Tarning J, Lindegardh N, Hanpithakpon W, et al. Artesunate/dihydroartemisinin pharmacokinetics in acute falciparum malaria in pregnancy: absorption, bioavailability, disposition and disease effects. Br J Clin Pharmacol. 2012;73:467-77.

28. Ahn JE, Karlsson MO, Dunne A, Ludden TM. Likelihood based approaches to handling data below the quantification limit using NONMEM VI. J Pharmacokinet Pharmacodyn. 2008;35:401-21.

29. Jusko WJ. Use of pharmacokinetic data below lower limit of quantitation values. Pharm Res. 2012;29:2628-31.

30. Bergstrand M, Karlsson MO. Handling data below the limit of quantification in mixed effect models. AAPS J. 2009;11:371-80.

31. Nadler SB, Hidalgo JH, Bloch T. Prediction of blood volume in normal human adults. Surgery. 1962;51:224-32.

32. Tran HT, White NJ, Nguyen TT, Nhu HT, Phung TD, Tarning J, et al. Estimation of the in vivo minimum inhibitory concentration of cipargamin in uncomplicated Plasmodium falciparum malaria. Antimicrob Agents Chemother. 2017;61:e01940-16.

33. White NJ. Antimalarial drug resistance. J. Clin Investig. 2004;113:1084-92.

34. Upton RN, Mould DR. Basic concepts in population modeling, simulation, and model-based drug development: part 3-Introduction to Pharmacodynamic Modeling Methods. CPT Pharmacometrics Syst Pharmacol. 2014;3:e88

35. Gisleskog P, Karlsson M, Beal S. Use of prior information to stabilize a population data analysis. J Pharmacokinet Pharmacodyn. 2002;29:473-505.

36. Lohy Das J, Dondorp AM, Nosten F, Phyo AP, Hanpithakpong W, Ringwald $P$, et al. Population pharmacokinetic and pharmacodynamic modeling of artemisinin resistance in Southeast Asia. AAPS J. 2017:19:1842-54.

37. Morris CA, Duparc S, Borghini-Fuhrer I, Jung D, Shin CS, Fleckenstein L. Review of the clinical pharmacokinetics of artesunate and its active metabolite dihydroartemisinin following intravenous, intramuscular, oral or rectal administration. Malar J. 2011:10:263

38. Tan B, Naik H, Jang IJ, Yu KS, Kirsch LE, Shin CS, et al. Population pharmacokinetics of artesunate and dihydroartemisinin following single-and multiple-dosing of oral artesunate in healthy subjects. Malar J. 2009;8:304

39. Van Acker K, Mommaerts M, Vanermen S, Meskens J, Heyden YV, PlaizierVercammen J. Chemical stability of artemisinin derivatives. Malar J. 2012;11(Suppl 1):P99

40. Talundzic E, Okoth SA, Congpuong K, Plucinski MM, Morton L, Goldman IF, et al. Selection and spread of artemisinin-resistant alleles in Thailand prior to the global artemisinin resistance containment campaign. PLoS Pathog. 2015;11:e1004789.

41. WHO Malaria Policy Advisory Committee Meeting Group. Minutes of the technical expert group (TEG) on drug efficacy and response. Geneva: World Health Organization; 2016.

42. Woodrow CJ, White NJ. The clinical impact of artemisinin resistance in Southeast Asia and the potential for future spread. FEMS Microbiol Rev. 2017:41:34-48.

43. van Schalkwyk DA, Sutherland CJ. Malaria resistance to non-artemisinin partner drugs: how to reACT. Lancet Infect Dis. 2015;15:621-3. 